\title{
The design of mechatronic system for gel electrophoresis
}

\author{
Apostolos Tsagaris ${ }^{1, *}$, Konstantinos Theodoridis ${ }^{1}$, Fotis Stergiopoulos ${ }^{1}$, Dimitrios Bechtsis ${ }^{1}$, \\ Nikolaos Nikolaidis ${ }^{1}$, Dimitrios Triantafyllidis ${ }^{1}$, Asterios Papaoikonomou ${ }^{2}$ and Anastasios \\ Filelis $^{2}$ \\ ${ }^{1}$ International Hellenic University (IHU), Department of Industrial Engineering \& Management, PO \\ Box 141, Sindos, Thessaloniki, 57400, Greece \\ ${ }^{2}$ Evresis S.A DA 12a Block:39b Industrial Area, 57400, Sindos, Thessaloniki
}

\begin{abstract}
The paper present the methodology to design and implement a mechatronic system for gel electrophoresis. It describes the steps towards the development of a prototype and presents its functions. The new automated system is characterized by high precision, small size and relatively low cost, thus achieving the introduction of a significant innovation in the area of medical and laboratory measurements.
\end{abstract}

\section{Introduction}

Electrophoresis is the phenomenon of the movement of chemical compounds due to the application of an electric field. The movement of substances within the electric field is directly related mainly to the applied electric charge and the molecular weight of the substances. Therefore, if an electric field $\mathrm{E}$ is applied to an electrophoresis device, the motion depends exclusively on the molecular weight of the substance, on a given substrate ( $\mathrm{pH}$, water content, temperature, etc.). For the electrophoresis process, it is necessary to use protocols for receiving and maintaining the sample, the characteristics of the electric field and the use of the appropriate substrate and the corresponding regulatory solutions.

The electrophoresis, as a process, is a simple technique, providing immediate results of high accuracy and low cost. It is used for the analysis of proteins in the blood serum but also in other biological fluids (e.g. urine, cerebrospinal fluid, saliva). Electrophoresis can be applied both as a research method and for clinical diagnosis of gammoglobinopathies, hemoglobinopathies, lipidemia, etc. As a diagnostic method, it provides clear quantitative (photometric evaluation of electrophoresis) and qualitative information for individual protein changes and contributes to the immediate and indirect diagnosis of pathological conditions, such as multiple myeloma, spinal cord disease, AIDS etc.

Nowadays the main technological methods for electrophoresis are two:

a) Electrophoresis with gel substrate such as agarose - natural polysaccharide (gel electrophoresis)

b) Capillary Electrophoresis

\footnotetext{
*Corresponding author: tsagaris@gmail.com
} 
Specificity as well as sensitivity play an important role for correct and reliable results. According to the international literature, agarose substrate electrophoresis provides higher selectivity $(98.9 \%)$ than capillary electrophoresis $[1,2]$

The typical steps of an agarose substrate electrophoresis process can be summarized as follows:

1. Sample taking and placing on an appropriate agarose substrate

2. Application of the electric field (constant conditions)

3. Drying of the processed sample

4. Staining and unstaining of the processed sample

5. Drying of the sample

6. Scanning using a computer

7. Results analysis

8. Printing and presentation of the results

\section{State of the art}

Technological developments are gradually evolving the process of electrophoresis. Today the commercial devices that exist are distinguished into manual and semi-automatic. In manual devices the transition from one stage of processing to another is done with human intervention while the semi-automatic ones do not include the part of scanning and exporting results to the device.

In [3], , researchers have developed a fully automated system for two-dimensional (2D) electrophoresis procedures. It is designated for rapid and highly reproducible protein analysis. The system can automatically complete, apart from situ protein detection, also isoelectric focusing (IEF), on-part protein staining and sodium dodecyl sulfate polyacrylamide gel electrophoresis (SDS-PAGE). For the evaluation the resultscomparison with a commercial minigel system has been performed. The proposed system achieved high-resolution despite shorter focusing time and smaller part dimensions. The resulting reproducibility was better or comparable to the performance of the minigel system. Complete 2D separation was achieved within $1.5 \mathrm{~h}$ [3].

In another case, a new device is described for the casting of many PAGE gels with improved practicality and performance due to the fact that the system contains four detachable components. This produces multiple gels in less time and more easily. The system is characterized by ease of construction, versatility and low cost that makes it competitive in the market [4]. Development of the Capillary Electrophoresis (CE) led to more efficient systems, like the microchip electrophoresis [5]. The microchip system provides a fast and effective analysis as it contains various microchannels that are made of glass and/or fused silica substrates with optimal thermal characteristics. The advantages of CE provided the opportunity to researchers to develop reliable, fast and economic fully automated systems [6] or semi-automated portable devices [7].

In [8] a ready-to-use device is proposed, which runs on the Agilent 2200 TapeStation instrument and provides a Quality Control reproducible method for analyzing the integrity and quantity of genomic DNA in combination with the ease of an automated system. The preparation of the required samples is minimal and the loading is automated. The results are in digital format and can be presented as a gel image, a data table and an electro-application viewer. Expert software gives the system a lot of potential, greatly increasing its functionality and usability [8].

Finally, according to a market survey, the company Sebia (www.sebia.com) holds a dominant position in the international electrophoresis market, while Beckman Coulter (www.beckmancoulter.com) also holds a significant share. However, there are other smaller companies in some countries that are mainly active in the automation of specific 
stages of electrophoresis, or in the manufacturing of devices for semi-automated processes, or finally in the production of consumables (film and gel). However, it is emphasized that even today, even the so-called "automatic" devices are not fully in the sense that they do not include a scanner and a computer for processing the film, and the final processing stage is always done in a separate computer with specialised software.

\section{The Design of mechatronic system}

As mentioned before, the aim of the paper is to present the design and manufacturing of a prototype of a new fully automated agarose gel electrophoresis device with significant reliability, small size, portability, and low cost advantages.

The steps to be followed for an automated electrophoresis process are summarized below (Fig. 1)

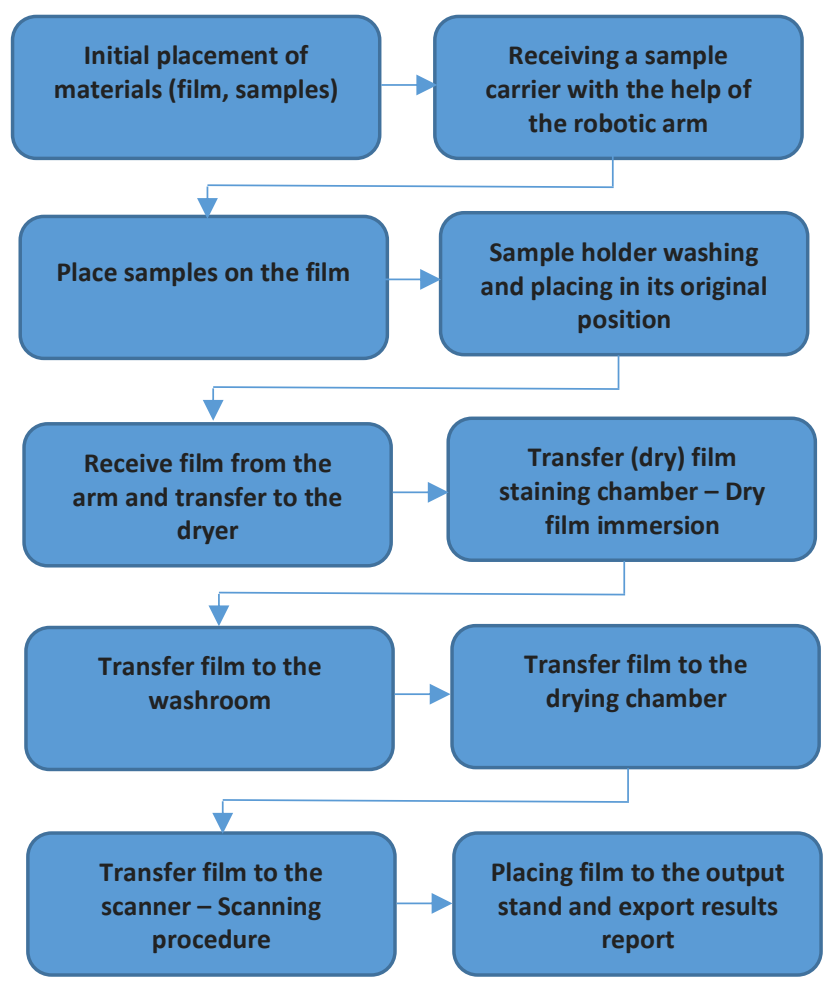

Fig. 1 - Steps for automated electrophoresis process

The construction methodology of the device performs the above steps and consists of four (4) basic phases:

1. In the first phase, the primary research is carried out for the analysis of the requirements, the $3 \mathrm{D}$ design of the individual parts and the analysis of the technical specifications for the electrical, electronic and mechanical parts of the device. The results of the preliminary study that have already been carried out and are used as the starting point.

2. In the second phase, the individual parts of the device are manufactured, as prototypes, for the implementation of mechanical components, arm and electronic and electrical parts, as well as firmware and software. The second phase can give feedback to the first phase to fine tune the technical specifications. 
3. In the third phase, pilot tests are performed, with the interconnection of the individual sections developed in the second phase. Pilot tests are divided into two stages:

I. Stage of creation of the original prototype, where the initial assembly and control of both the individual parts and a first version of the device are performed. In addition, the operation of the individual parts is studied and issues of functionality and needs of redesign / improvements of parts of the device are highlighted.

II. Stage of creation of the final prototype, after receiving the feedback from the original prototype. Specifically, optimization and redesign will be performed in order to reduce the volume of the device and to adjust external covers. In the second stage, a substantial redesign of the original is carried out and all the problems presented during the first stage of construction are analyzed. Finally, all assembly procedures and synchronized hardware and software tests are performed to build the final and fully functional prototype.

The third phase may re-adjust the individual implementations of the second phase if necessary.

4. In the fourth phase, the study of the exploitation of the research results takes place. This phase is implemented immediately after the implementation of the standard and includes an exploitation study of the results, the investigation of possible patent results, the creation of publications and the development of a market promotion plan.

The following figure (fig. 2) shows the methodology.

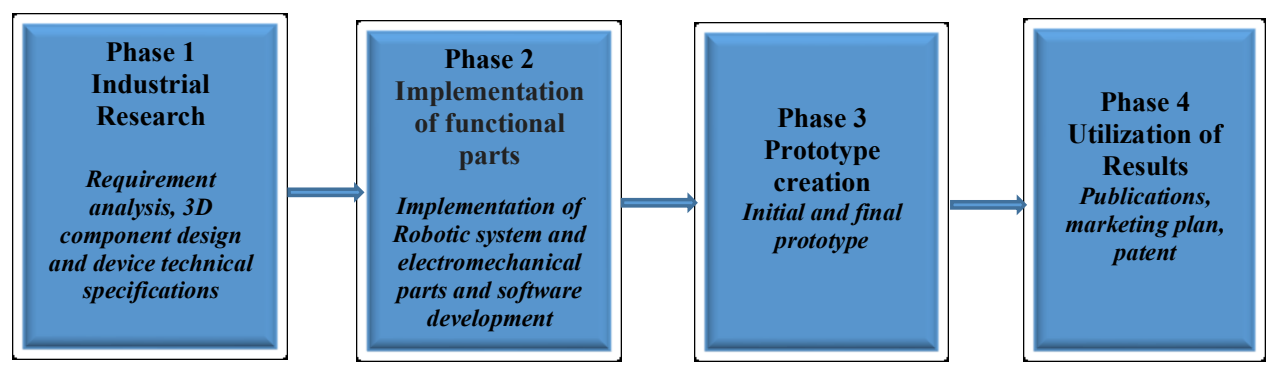

Fig. 2 - Implementation methodology

With the proposed methodology, the conditions for success will be created, as in each phase, possible errors will be highlighted and corrected in the next stage.

\section{System Description}

The electrophoresis mechatronic system consists of different subsystems which contribute to the necessary actions to complete the electrophoresis process. Emphasis was given on the design of the special parts and especially on the moving parts trying to incorporate many elements of intelligence into the mechatronic system. In the following figure (fig. 3) the main parts of the mechatronic system are shown which include: (a) a tube drawer system, (b) a bar code scanner, (c) a wash tank, (d) a sample carrier, (e) an electrophoresis chamber, (f) a robotic arm/gripper, (g) a staining-unstaining chamber, (h) a drying chamber, (i) a frame/film manipulator, (j) a camera analysis chamber. 


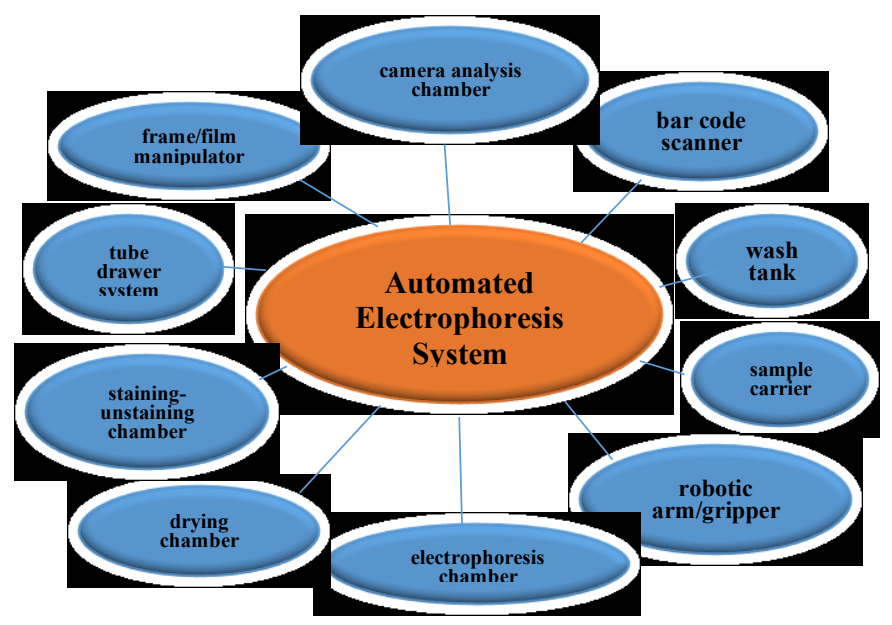

Fig. 3 - System configuration

The main part of the automatic electrophoresis machine is the mechatronic system (Fig. 4) that performs the sampling and movement routines of the film frame at the various stages of the electrophoresis process. It has the ability to move on two axes of motion in XX and ZZ. It has a properly designed clamp that has the ability to open and close in order to grab and hold components like a robotic end effector. The Closing of the end effector is ensured with a suitable sensor while the opening is performed with time calculated after the closing. The movement on the axes is limited by appropriate start and stop sensors.
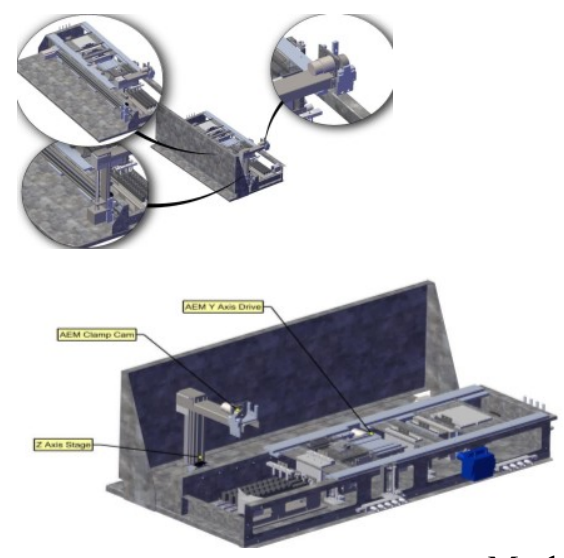

Fig. 4 - Mechatronic system

The XX axis of movement have a movement accuracy of $0.1 \mathrm{~mm}$ and is able to accelerate the system with $0.3 \mathrm{~m} / \mathrm{sec} 2$ up to a maximum speed of $0.7 \mathrm{~m} / \mathrm{sec}$. Two high-precision linear guides were used to reduce oscillations during acceleration and deceleration. The motor drivers and individual components such as position sensors were mounted on specially treated aluminum plate made of $6063 \mathrm{~T} 6$ material to achieve the necessary level of tolerance and parallelism thus minimizing losses and reducing the system's rolling resistance. The vertical axis of movement ZZ has an accuracy of $0.05 \mathrm{~mm}$ and accelerates to $0.1 \mathrm{~m} / \mathrm{sec} 2$. The high accuracy is crucial since the samples must be deposited in the agarose film without the screw of the sample carrier entering the substrate. Due to the 
increased required accuracy, the solution of the drive screw has been selected, through which high accuracy can be achieved by limiting the maximum speed.

\section{Conclusion}

An innovative fully automatic electrophoresis mechatronic system has been designed and manufactured that can perform electrophoresis processes on precast gel agarose films. The operation of the system was evaluated as well as the robustness of the machine by performing a complete electrophoresis process that includes protein analysis. The results showed high accuracy in operation and efficiency in completing the different phases of the electrophoresis process. An additional feature is the speed of execution of the process that helps the productivity of the system.

This research has been co-financed by the European Regional Development Fund of the European Union and Greek national funds through the Operational Program Competitiveness, Entrepreneurship and Innovation, under the call RESEARCH - CREATE - INNOVATE (project code: T1EDK-02403)

\section{References}

1. B. Lissoir, P. Wallemacq, D.Maisin, Ann Biol Clin (Paris).61, 557-562. (2003)

2. Ch. R. McCudden, S. P. Mathews, Sh. A. Hainsworth, J. F. Chapman, C. A. HammettStabler, M. S. Willis and D. G. Grenache, Am J Clin Pathol, 129:451-458 458 (2008)

3. A. Hiratsuka, H. Kinoshita, Y. Maruo, K Takahashi, S. Akutsu, Ch. Hayashida, K. Sakairi, K. Usui, K. Shiseki, H. Inamochi, Y. Nakada, K. Yodoya, I. Namatame, Y. Unuma, M. Nakamura, K. Ueyama, Y. Ishii, K. Yano, K. Yokoyama, 79 (15), 57305739 (2007)

4. P. Maurye, A. Basu, T. K. Bandyopadhyay, J. K. Biswas, B. P. Mohanty, ELECTROPHORESIS 201738 (16), 2060-2068 (2017)

5. B. C. Durney, C. L. Crihfield, L. A. Holland, Analytical and Bioanalytical Chemistry, 407(23), 6923-6938 (2015)

6. J. Bodnar, L. Hajba, A. Guttman, ELECTROPHORESIS, 37(23-24), 3154-3159 (2016)

7. T. A. Nguyen, T. N. Pham, T. T. Doan, T. T. Ta, J. Saiz, T. Q. Nguyen, T. D. Mai, J Chromatogr A, 1360, 305-311 (2014)

8. D. Rabiller, D. McDade-Walker, A. Padmanaban, R. Salowsky, M. Gassmann, J Biomol Tech 24(Suppl): S55 (2013) 